\title{
MEK inhibitor enhances sensitivity to chemotherapeutic drugs in multidrug resistant hepatocellular carcinoma cells
}

\author{
QINGLIANG MENG $^{1 *}$, XIAOQI HE ${ }^{2 *}$, GUANGWEI XIE $^{1}$, QINGZHONG TIAN $^{1}$, \\ XIAOGANG SHU ${ }^{3}, \mathrm{JIN} \mathrm{LI}^{1}$ and YONG XIAO \\ ${ }^{1}$ Department of Oncological Surgery, Xuzhou Central Hospital, Xuzhou, Jiangsu 221009; \\ Departments of ${ }^{2}$ Obstetrics and Gynecology and ${ }^{3}$ Gastrointestinal Surgery, Union Hospital, \\ Tongji Medical College, Huazhong University of Science and Technology, Wuhan, Hubei 430022, P.R. China
}

Received September 2, 2015; Accepted January 6, 2017

DOI: $10.3892 / \mathrm{ol} .2017 .6494$

\begin{abstract}
The aim of the present study was to investigate the association between the mitogen-activated protein kinase (MAPK) signal transduction pathway and multidrug resistance in hepatocellular carcinoma cells. A Cell Counting Kit-8 assay was used to determine the drug sensitivity of HepG2 and HepG2/ADM hepatocellular carcinoma cell lines in combination with the MAPK/extracellular-signal-regulated kinase kinase (MEK) inhibitor U0126. Flow cytometry was used to analyze the rate of apoptosis. The reverse transcription-quantitative polymerase chain reaction (RT-qPCR) was used to determine P-glycoprotein (P-gp) and multidrug resistance-associated protein 1 (MRP1) mRNA expression following treatment with various concentrations of U0126. P-gp and MRP1 expression levels were measured using Western blot analysis. The half-maximal inhibitory concentration was markedly decreased in combination with U0126. RT-qPCR results demonstrated that the expression of multidrug resistance 1 (MDR1) and MRP1 in HepG2/ADM cells was increased 5.37- and 6-14-fold compared with that in HepG2 cells. Furthermore, the expression levels in HepG2/ADM cells were decreased following U0126 treatment in a dose-dependent manner. The expression of P-gp and MRP1 in HepG2/ADM cells was increased 2.68- and 2.76-fold compared with that in HepG2 cells. Furthermore, the expression levels in HepG/ADM cells were decreased following U0126 treatment in a dose-dependent manner. The results of the present study
\end{abstract}

Correspondence to: Professor Yong Xiao, Department of Gastrointestinal Surgery, Union Hospital, Tongji Medical College, Huazhong University of Science and Technology, 1277 Jiefang Avenue, Wuhan, Hubei 430022, P.R. China

E-mail: xydoc@aliyun.com

*Contributed equally

Key words: multidrug resistance, hepatocellular carcinoma, P-glycoprotein, mitogen-activated protein kinase/extracellularsignal-regulated kinase kinase indicate that the MEK inhibitor U0126 enhances sensitivity to chemotherapeutic drugs by downregulating P-gp and MRP1 expression in resistant hepatocellular carcinoma cells. The combination of MEK inhibitor and conventional chemotherapeutic drugs may provide novel therapeutic prospects for the treatment of drug-resistant hepatocellular carcinoma.

\section{Introduction}

Liver cancer in males is the fifth most frequently diagnosed type of cancer worldwide; however, it is the second most frequent cause of cancer mortality. In females, it is the seventh most commonly diagnosed type of cancer and the sixth leading cause of cancer mortality (1). An estimated 748,300 cases of liver cancer were newly diagnosed and 695,900 cancer mortalities occurred worldwide in 2008 , with $50 \%$ of these cases and mortalities estimated to have occurred in China (2). The majority of patients with hepatopathy in China suffer as a result of hepatitis B cirrhosis; furthermore, the disease is typically diagnosed late, opportunities for excision are limited and sensitivity to radiotherapy is poor, therefore the majority of cases are treated using traditional chemotherapy (3). However, liver cancer exhibits poor sensitivity to chemotherapeutic drugs, and the side effects are not ideal; in particular, resistance to doxorubicin [Adriamycin (ADM)] in the treatment of liver cancer is $<80 \%$ (4). The poor sensitivity to chemotherapeutic drugs is associated with natural drug resistance and acquired multidrug resistance (MDR) in liver cancer (4). A number of drugs exist that are able to reverse the MDR of liver cancer; however, they exhibit increased toxicity and a limited effect. Therefore, the development of a novel drug to reverse MDR is of key importance for the treatment of liver cancer.

MDR describes the property of malignant cells exhibiting resistance to a number of chemotherapeutic drugs of distinct structure and mechanism of action. MDR is a key mechanism used by tumor cells to resist chemotherapeutic drugs. A number of factors may result in MDR, including the drug-efflux pump mechanism of drug resistance proteins, including $\mathrm{P}$-glycoprotein (P-gp), MDR-associated protein (MRP) and lung resistance-related protein (LRP), mutations in DNA topoisomerase and DNA repair abnormality (5). The drug efflux pump mediated by drug resistance proteins, 
including P-gp and MRP, is the primary molecular mechanism of MDR in tumor cells (6).

Cellular signal transduction pathways serve a role in malignant tumors, in which abnormalities in the mitogen-activated protein kinase (MAPK) signaling pathway activated by growth factors such as epidermal growth factor have been associated with the growth, proliferation and invasion of malignant tumors, and resistance to chemotherapeutic drugs $(7,8)$. Mammals possess three classical MAPK signaling pathways, in which the Raf proto-oncogene serine/threonine protein kinase (Raf)/MAPK/extracellular-signal-regulated kinase $(E R K)$ kinase $(\mathrm{MEK}) / \mathrm{ERK}$ signal transduction pathway is the most well-researched $(9,10)$. It has been demonstrated previously that ADM in lymphocytoma B is able to induce the expression of P-gp to generate drug resistance by activating MAPK/ERK (11). Abrams et al (12) demonstrated that a targeted drug aimed at Raf/MEK/ERK signal transduction pathway was able to reverse the drug resistance of leukemia drug resistance cells and enhance the sensitivity of tumor-resistance chemotherapeutic drugs. Katayama et al (13) demonstrated that the MEK inhibitor U0126 was able to downregulate the expression of endogenous P-gp of SW620-14 cells and the expression of exogenous P-gp of MCF-7/MDR and MDA-MB-231/MDR to enhance the anti-tumor activity. However, the association between MAPK and MDR in primary liver cancer remains unclear.

The aim of the present study was to elucidate the interaction between the MAPK signaling pathway and ATP-binding cassette $(\mathrm{ABC})$ protein expression in hepatocellular carcinoma (HCC). A selective inhibitor of MEK activity (U0126) was used to investigate the effects on P-gp and MRP1 protein expression.

\section{Materials and methods}

Cell lines and reagents. The ADM, VCR, 5-FU and MMC were purchased from Zhejiang Hisun Pharmaceutical Co., Ltd (Taizhou City, Zhejiang Province). The HepG2 and HepG2/ADM cell lines were purchased from Beijing North Carolina Souren Biotechnology Research Institute, (Beijing, China). U0126 was purchased from (Selleck Chemicals, Houston, TX, USA), and the RPMI 1640 mediun and fetal bovine serum were purchased from (Hyclone; GE Healthcare, Logan, UT, USA).

Cell culture. The ADM-resistant human HCC cell line HepG2/ADM was cultured in RPMI-1640 medium containing $10 \%$ fetal bovine serum, 100 units $/ \mathrm{ml}$ penicillin and $100 \mathrm{mg} / \mathrm{ml}$ streptomycin (Hyclone; GE Healthcare, Logan, UT, USA) at $37^{\circ} \mathrm{C}$ in an incubator with $5 \% \mathrm{CO}_{2}$ and $95 \%$ humidity. ADM $(0.4 \mathrm{nmol} / \mathrm{ml})$ was added to the culture medium to maintain the drug resistance of HepG2/ADM cells. The HepG2 cells also cultured in this way, without adding ADM.

Investigation of drug resistance in HepG2/ADM cells and sensitivity to chemotherapeutic drugs. A total of $0.2 \mathrm{ml}$ HepG2/ADM and HepG2 cells in the exponential growth phase were inoculated in 96-well plates at a density of $1 \times 10^{5} /$ well). Following incubation at $37^{\circ} \mathrm{C}$ and $5 \% \mathrm{CO}_{2}$ for $24 \mathrm{~h}$, the supernatant was discarded and fresh medium containing chemotherapeutic drugs (ADM, VCR, 5-FU and MMC) at various concentrations (ADM; 0, 0.1, 1, 10, 100 and $1,000 \mathrm{mg} / \mathrm{ml}$; VCR, 5-FU and MMC; 0, 2, 4, 8, 16, $32 \mathrm{mg} / \mathrm{l})$ was added into culture plates. Following incubation for $24 \mathrm{~h}$ $37^{\circ} \mathrm{C}$ and $5 \% \mathrm{CO}_{2}$, the supernatant was discarded, $10 \mu \mathrm{l}$ Cell Counting Kit-8 (CCK-8) was added to each well and cells were cultured for a further $4 \mathrm{~h}$ under the same conditions, in the absence of drugs. A microplate reader was used to measure the absorbance of each well at $450 \mathrm{~nm}$. The half-maximal inhibitory concentration $\left(\mathrm{IC}_{50}\right)$ was calculated to determine the resistance indices of HepG2/ADM and HepG2 cells to the chemotherapeutic drugs used.

Determination of the effect of U0126 at various concentrations on the apoptotic rates of HepG2/ADM cells. HepG2/ADM cells in exponential growth phase were seeded in 6-well plates $\left(1 \times 10^{5}\right.$ cells/well) and incubated for $24 \mathrm{~h}$. The supernatant was discarded, medium containing various U0126 concentrations $(0,10,20$ and $40 \mu \mathrm{mol} / \mathrm{l})$ was added into the corresponding wells and the cells were incubated for $48 \mathrm{~h}$. EDTA-free trypsin $(0.25 \%)$ was used to detach cells and cells were divided into groups. The detached cells were washed twice using centrifugation at $503.1 \mathrm{x} \mathrm{g}$ for $5 \mathrm{~min}$ at $37^{\circ} \mathrm{C}$. An Annexin V/propidium iodide (PI) apoptosis detection kit. A Cell Proliferation and Cytotoxicity Assay Kit (Nanjing KeyGen Biotech. Co., Ltd., Nanjing, China) was used to determine the apoptosis rate of HepG2/ADM cells, according to the manufacturer's protocol. Subsequently, cells were harvested and washed twice, and resuspended in $1 \mathrm{X}$ binding buffer (Beyotime Institute of Biotechnology, Haimen, China) at a concentration of $1 \times 10^{6}$ cells $/ \mathrm{ml}$. A $100 \mu \mathrm{l}$ cell suspension was stained with $5 \mu \mathrm{l}$ Annexin V-fluorescein isothiocyanate and $5 \mu \mathrm{l}$ PI at room temperature for $15 \mathrm{~min}$ in the dark, prior to analyzing the cells using flow cytometry.

Reverse transcription-quantitative polymerase chain reaction (RT-qPCR). The expression of MDR1 and MRP1 mRNA was analyzed using RT-qPCR (14), with human $\beta$-actin (284bp; sequence: Forward 1379: 5'-AGCGAGCATCCC CCAAAGTT-3'; reverse, 1663: 5'-GGGCACGAAGGCTCA TCATT-3') as an internal control. TRIzol ${ }^{\circledR}$ (Qingdao Jisskang Biotechnology Co., Ltd., Qingdao, China) (1 ml) was added to the harvested cells in a microcentrifuge tube and agitated until evenly mixed. Chloroform $(0.2 \mathrm{ml})$ was added to the mixture, and the microcentrifuge tube was inverted a number of times and left to stand for $5 \mathrm{~min}$ at room temperature. Subsequently, the tube was centrifuged at 4,024.8 $\mathrm{x} g$ for $15 \mathrm{~min}$ at $4^{\circ} \mathrm{C}$. The upper aqueous phase $(\sim 400 \mu \mathrm{l})$ was transferred into a new $1.5 \mathrm{ml}$ microcentrifuge tube and $400 \mu \mathrm{l}$ propan-2-ol was added. The contents of the tube were mixed evenly and left to stand for $10 \mathrm{~min}$ at room temperature. The mixture was centrifuged at $4,024.8 \mathrm{x} \mathrm{g}$ for $10 \mathrm{~min}$ at $4^{\circ} \mathrm{C}$. The supernatant was discarded, and the precipitate was washed three times with $70 \%$ ice-cold ethanol and air-dried for between 5 and $10 \mathrm{~min}$. Diethylpyrocarbonate in $\mathrm{H}_{2} \mathrm{O}$ $(20 \mu \mathrm{l})$ was added to the tube to dissolve the precipitate. The quality and concentration of RNA was determined using a spectrophotometer. [RNA concentration=OD260x40 $\mu \mathrm{g} / \mathrm{ml}$ x Dilution Multiple (4)] cDNA was synthesized from total mRNA using a PrimeScript RT reagent kit. Following 
first-strand cDNA synthesis $\left(50^{\circ} \mathrm{C}\right.$ for $2 \mathrm{~min}, 95^{\circ} \mathrm{C}$ for $10 \mathrm{~min}, 95^{\circ} \mathrm{C}$ for $30 \mathrm{sec}$, then $60^{\circ} \mathrm{C} 30 \mathrm{sec}$ for 40 cycles). PCR amplification using the Ex Taq ${ }^{\mathrm{TM}}$ kit (Takara Biotechnology, Co., Ltd., Dalian, China) was conducted using the following primers: MDR1: Forward, 5'TGATTGCATTTG GAGGACAA 3', reverse 5'CCAGAAGGCCAGAGCATA AG 3'; MRP1, forward, 5'AGGTGGACCTGTTTCGTGAC 3'; reverse, 5'CCTGTGATCCACCAGAAGGT 3'. DNA was amplified using the following protocol: $2 \mathrm{~min}$ at $50^{\circ} \mathrm{C}$ and $10 \mathrm{~min}$ at $95^{\circ} \mathrm{C}$, followed by 40 cycles of $95^{\circ} \mathrm{C}$ for $30 \mathrm{sec}$ and $60^{\circ} \mathrm{C}$ for $30 \mathrm{sec}$. Oligonucleotides and reagents for the PCR assay were purchased from Nanjing Kingsley Biological Technology Co., Ltd. (Nanjing, China). Data were analyzed with Sequence Detector software (version 1.9; Applied Biosystems; Thermo Fisher Scientific, Inc., Waltham, MA, USA). The mean $\mathrm{Cq}$ value for duplicate measurements was used to detect the expression of target gene normalized to the housekeeping gene $\beta$-actin, which was used as an internal control.

Western blot analysis. The expression of drug-resistant P-gp and MRP1 were analyzed using western blot analysis. A total of $1.2 \times 10^{6} \mathrm{HepG} 2 / \mathrm{ADM}$ and HepG2 cells in exponential growth phase were inoculated into 6-well plates. Cells were harvested following treatment with U0126 and 10,20 and $40 \mu \mathrm{mol} / 1 \mathrm{ADM}$ for $48 \mathrm{~h}$ at $37^{\circ} \mathrm{C}$, and subsequently washed twice with PBS. A radioimmunoprecipitation assay lysis buffer $(50 \mathrm{mM}$ Tris ( $\mathrm{pH} 7.4$ ), 150mM NaCl, $1 \%$ Triton $\mathrm{X}-100,1 \%$ sodium deoxycholate, $0.1 \%$ SDS, sodium orthovanadate, sodium fluoride, EDTA and leupeptin; Beyotime Institute of Biotechnology) was used to homogenize cells. Following detection of the protein concentration using a bicinchoninic acid assay (Beyotime Institute of Biotechnology), $75 \mu \mathrm{g}$ of each sample was separated using SDS-PAGE (8\% gel). Following separation of target proteins from total protein determined according to size based on that of the pre-stained markers, electrophoresis was stopped. Following removal of the hydrogel, the target protein bands were excised, washed with distilled water and electrotransferred onto polyvinylidene fluoride membranes. The membrane was blocked with TBS-5\% Tween-20 (TBST) containing $5 \%$ skimmed milk powder at room temperature for $2 \mathrm{~h}$ and incubated at $4^{\circ} \mathrm{C}$ overnight with the primary antibodies directed against the following: MRP1 (cat. no. GTX116046; dilution, 1:1,000; GeneTex International Corporation, Hsinchu, Taiwan) and P-Glycoprotein (cat. no. GTX108354; dilution, 1:1,000; GeneTex International Corporation). Following washing with TBST, the membrane was incubated at $37^{\circ} \mathrm{C}$ with horseradish peroxidase-conjugated secondary antibody (cat. no. BA1054; Beyotime Institute of Biotechnology) for $2 \mathrm{~h}$. Following a further wash with TBST, labeled proteins were visualized using an Film Development enhanced chemiluminescence kit (cat. no. P0020; Wuhan Booute Biotechnology Co, Ltd., Wuhan, China) on high-performance chemiluminescence film according to the manufacturer's protocol.

Statistical analysis. Statistical analysis was performed using SPSS software (version 19.0; IBM SPSS, Armonk, NY, USA). Results were presented as the mean \pm standard deviation of three replicates. A one-way analysis of variance was applied for comparison among multiple sets of data. The multiple
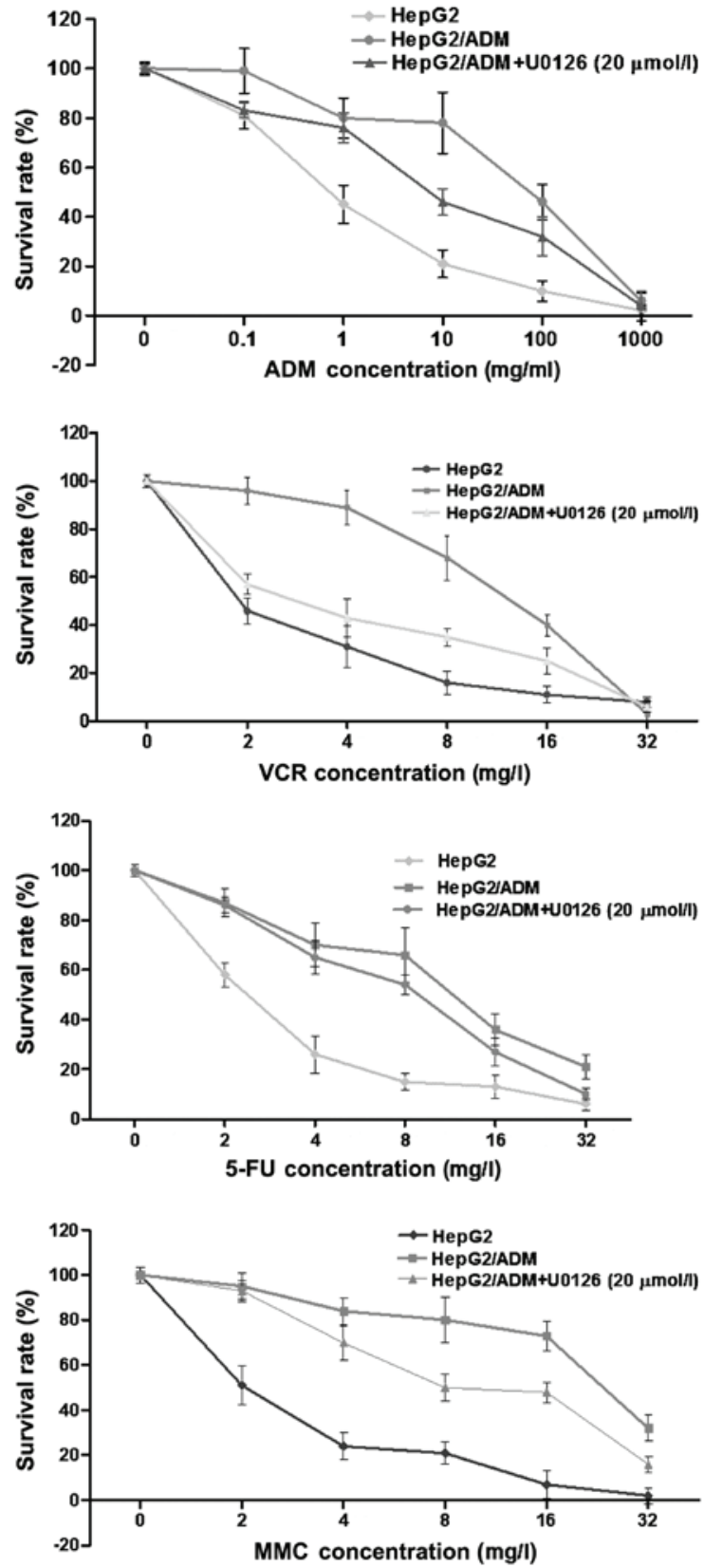

Figure 1. Sensitivity of HepG2 and HepG2/ADM cells to various chemotherapeutic drugs, and the effect of U0126. ADM, Adriamycin; VCR, vincristine; 5-FU, 5-fluorouracil; MMC, mitomycin.

comparison between the groups was performed using the Student-Newman-Keuls method. $\mathrm{P}<0.05$ was considered to represent a statistically significant difference.

\section{Results}

HepG2/ADM cells exhibit increased resistance to chemotherapeutic drugs compared with Hep 2 cells, which is decreased by U0126 treatment. HepG2 and HepG2/ADM cells were incubated with increasing doses of ADM, VCR, 5-FU and MMC to determine their sensitivities to each chemotherapeutic drug (Fig. 1). The $\mathrm{IC}_{50}$ values for each drug were significantly increased in HepG2/ADM cells compared with HepG2 cells $(\mathrm{P}<0.01$; Table I; Fig. 2), demonstrating that HepG2/ADM cells exhibited drug resistance to ADM and the other chemotherapeutic drugs. 
Table I. Determination of $\mathrm{IC}_{50}$ values and resistance indices of various anticancer drugs.

\begin{tabular}{lcccc}
\hline & \multicolumn{3}{c}{$\mathrm{IC}_{50}$} & \\
\cline { 2 - 4 } Drug & HepG2 & HepG2/ADM & HepG2/ADM+U0126 & Resistance index \\
\hline ADM (g/l) & $1.065 \pm 0.105$ & $29.57 \pm 1.756^{\mathrm{a}}$ & $5.796 \pm 0.143^{\mathrm{b}}$ & 27.77 \\
VCR (mg/l) & $1.471 \pm 0.560$ & $9.650 \pm 0.912^{\mathrm{a}}$ & $3.250 \pm 0.579^{\mathrm{b}}$ & 6.560 \\
5 -FU (mg/l) & $1.958 \pm 0.904$ & $10.28 \pm 1.012^{\mathrm{a}}$ & $6.930 \pm 0.315^{\mathrm{b}}$ & 5.250 \\
MMC (mg/l) & $2.117 \pm 0.406$ & $23.36 \pm 0.869^{\mathrm{a}}$ & $10.27 \pm 0.751^{\mathrm{b}}$ & 11.03 \\
\hline
\end{tabular}

Results are presented as the mean \pm standard deviation. $\mathrm{IC}_{50}$, half-maximal inhibitory concentration; ADM, Adriamycin; VCR, vincristine; 5-FU, 5-fluorouracil; MMC, mitomycin. ${ }^{\mathrm{a}} \mathrm{P}<0.01$ vs. HepG2 cells. ${ }^{\mathrm{b}} \mathrm{P}<0.01$ vs. HepG2/ADM cells.

In order to investigate whether U0126 was able to enhance the chemotherapeutic effects of the drugs, HepG2/ADM cells were pre-treated with U0126 (20 $\mu \mathrm{mol} / \mathrm{l})$ for $24 \mathrm{~h}$, followed by the drugs for a further $48 \mathrm{~h}$. Cell viability was determined using a CCK-8 kit. Treatment with U0126 (20 $\mu \mathrm{mol} / \mathrm{l})$ on HepG2/ADM cells led to a significant decrease in its $\mathrm{IC}_{50}$ values for all four drugs compared with that of untreated HepG2/ADM cells $(\mathrm{P}<0.01$; Table I; Fig. 2$)$, demonstrating that U0126-treated HepG2/ADM cells exhibited increased sensitivity to ADM, VCR, 5-FU and MMC compared with untreated HepG2/ADM cells.

U0126 induces apoptosis of HepG2/ADM cells in a dose-dependent manner. Although U0126 was demonstrated to increase the sensitivity of HepG2/ADM cells to the chemotherapeutic drugs ADM, VCR, 5-FU and MMC, the underlying molecular mechanisms for the effect of U0126 remain unclear. To investigate whether this treatment was able to induce apoptosis in HepG2/ADM cells, flow cytometry was used to determine the proportion of apoptotic cells induced by treatment with U0126. The results demonstrated that U0126 induced an increased incidence of apoptosis of HepG2/ADM cells compared with untreated HepG2/ADM control cells (Fig. 3), and the increase in apoptosis was identified to be dose-dependent (Fig. 4).

U0126 inhibits MDR1 and MRP1 mRNA expression in HepG2/ADM cells. In order to identify the underlying molecular mechanism for the U0126-mediated enhancement of drug sensitivity in HepG2/ADM cells, the effects of U0126 on MDR1 and MRP1 mRNA expression in HepG2/ADM cells were investigated using RT-qPCR. RT-qPCR analysis demonstrated that U0126 significantly downregulated MDR1 and MRP1 mRNA expression in a dose-dependent manner $(\mathrm{P}<0.05$; Fig. 5). MDR1 mRNA expression in the drug-resistant HepG2/ADM cells was significantly increased (5.37-fold) compared with that of drug-sensitive HepG2 parental cells (P<0.05; Fig. 5). Similarly, MRP1 mRNA expression in the drug-resistant HepG2/ADM cells was significantly increased (6.14-fold) compared with that of drug-sensitive HepG2 parental cells $(\mathrm{P}<0.05$; Fig. 5). These results indicated that a potential underlying molecular mechanism for the U0126-mediated enhancement of drug sensitivity in HepG2/ADM cells was the downregulation of MDR1 and MRP1 mRNA expression.

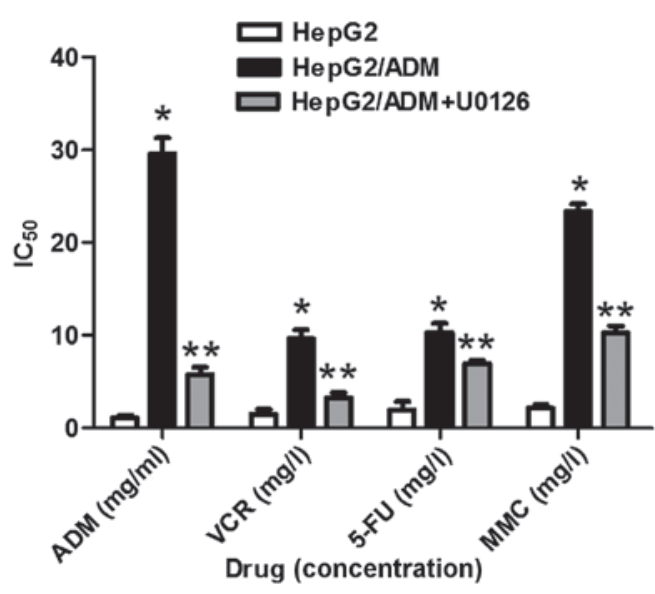

Figure 2. Mean $\mathrm{IC}_{50}$ values of various chemotherapeutic drugs in HepG2 and HepG2/ADM cells alone or in combination with U0126. Results are presented as the mean \pm standard deviation $(n=3) .{ }^{*} \mathrm{P}<0.01$ vs. HepG2 cells. ${ }^{* *} \mathrm{P}<0.01$ vs. HepG2/ADM cells. $\mathrm{IC}_{50}$, half-maximal inhibitory concentration; ADM, Adriamycin; VIN, vincristine; 5-FU, 5-fluorouracil; MMC, mitomycin.

U0126 induces P-gp and MRP1 protein downregulation. Anticancer drugs exhibit limited activity and a poor response when used in the treatment of drug-resistant cells. P-gp is the factor most frequently involved in MDR (15). The effects of U0126 on P-gp protein levels in drug-sensitive HepG2 parental cells and drug-resistant HepG2/ADM cells were investigated. Following a $24 \mathrm{~h}$ incubation with U0126, a concentration-dependent decrease in P-gp levels was detected in HepG2/ADM cells, with the decrease detectable at concentrations of U0126 as low as $10 \mu \mathrm{mol} / \mathrm{l}$. The relative expression of P-gp protein in the drug-resistant HepG2/ADM cells was increased 2.68-fold compared with that in drug-sensitive HepG2 parental cells $(0.5857 \pm 0.0235$ vs. $0.2183 \pm 0.0273$, respectively; Fig. 6). Furthermore, a number of recent studies have demonstrated the importance of functional MRP1 in response to individual pathway inhibitors (16). For this reason, MRP1 protein expression was determined in HepG2 and HepG2/ADM cell lines, following $24 \mathrm{~h}$ treatment with increasing concentrations of U0126. In the cell lines, a concentration-dependent decrease in MRP1 protein expression levels was demonstrated. The relative expression of MRP1 protein in the drug-resistant HepG2/ADM cells was increased 2.76-fold compared with that of drug-sensitive HepG2 parental cells $(0.5953 \pm 0.0271$ vs. 
A
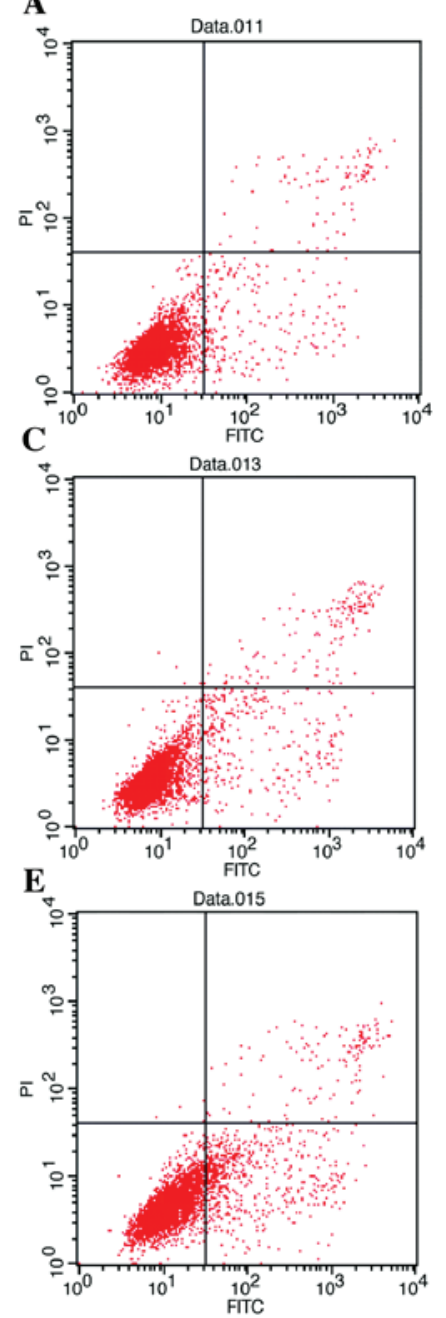

B
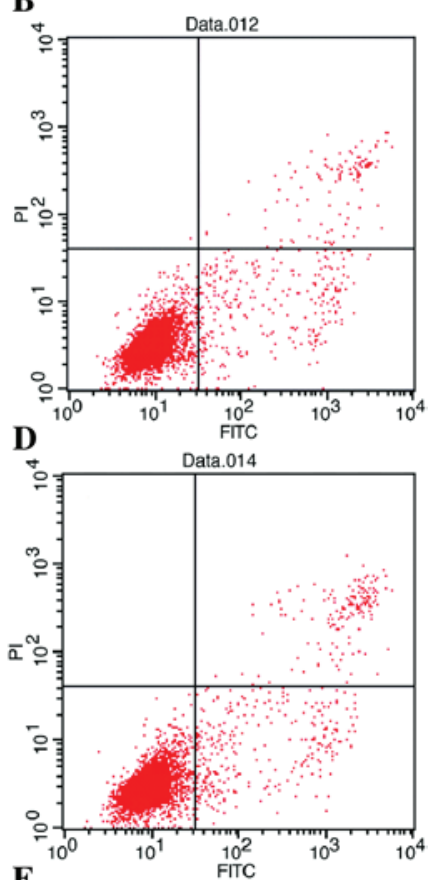

$\mathbf{F}$

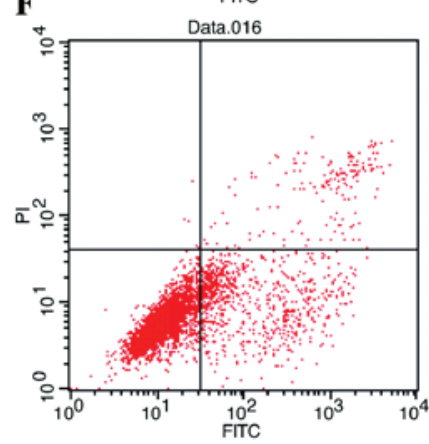

Figure 3. Flow cytometric analysis of apoptosis of HepG2/ADM cells. (A) Untreated HepG2/ADM cells (early apoptosis, $4.88 \%$; late apoptosis, $1.51 \%)$. (B) HepG2/ADM cells treated with ADM $(0.4 \mu \mathrm{mol} / \mathrm{l})$ after $48 \mathrm{~h}$ (early apoptosis, 5.49\%; late apoptosis, $2.13 \%$ ). (C) HepG2/ADM cells treated with U0126 (40 $\mu \mathrm{mol} / \mathrm{l}$ ) (early apoptosis, 6.67\%; late apoptosis, $3.12 \%)$. (D) HepG2/ADM cells treated with ADM $(0.4 \mu \mathrm{mol} / \mathrm{l})$ and U0126 (10 $\mu \mathrm{mol} / \mathrm{l}$ ) (early apoptosis, $10.75 \%$; late apoptosis, $3.53 \%$ ). (E) HepG2/ADM cells treated with ADM $(0.4 \mu \mathrm{mol} / 1)$ and $\mathrm{U} 0126(20 \mu \mathrm{mol} / \mathrm{l})$ (early apoptosis, 16.83\%; late apoptosis, 2.93\%). (F) HepG2/ADM cells treated with ADM $(0.4 \mu \mathrm{mol} / 1)$ and U0126 (40 $\mu \mathrm{mol} / \mathrm{l})$ (early apoptosis, $21.15 \%$; late apoptosis, 4.37\%). ADM, Adriamycin; FITC, fluorescein isothiocyanate; PI, propidium iodide.

$0.2153 \pm 0.0341$, respectively; Fig. 6 ). These results indicated that U0126 enhances the sensitivity of HepG2/ADM cell to chemotherapeutic drugs via downregulated expression of P-gp and MRP1 protein.

\section{Discussion}

MAPKs serve a critical role in the transduction of extracellular signals into cells to regulate differentiation, proliferation and apoptosis. Tumor cells exhibit resistance to the chemotherapeutic drugs to which they are exposed; however, tumor cells also exhibit resistance to chemotherapeutic drugs with a molecular structure that has not been encountered previously as well as to chemotherapeutic drugs that exert their effects via a different mechanism of action (17). HepG2/ADM cells

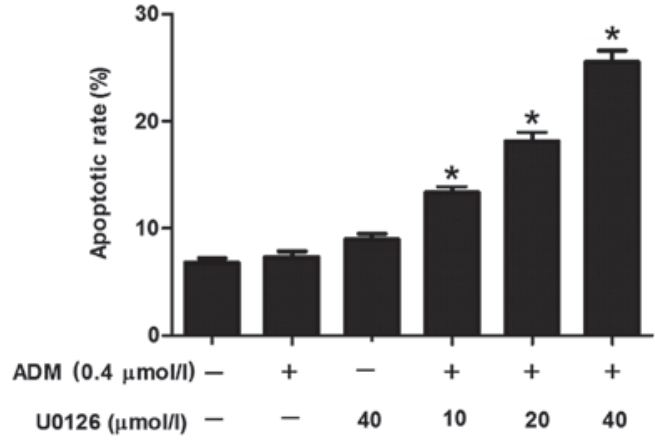

Figure 4. Effect of U0126 on apoptosis of HepG2/ADM. "P<0.05 vs. cells treated with chemotherapeutic drugs alone. ADM, Adriamycin.

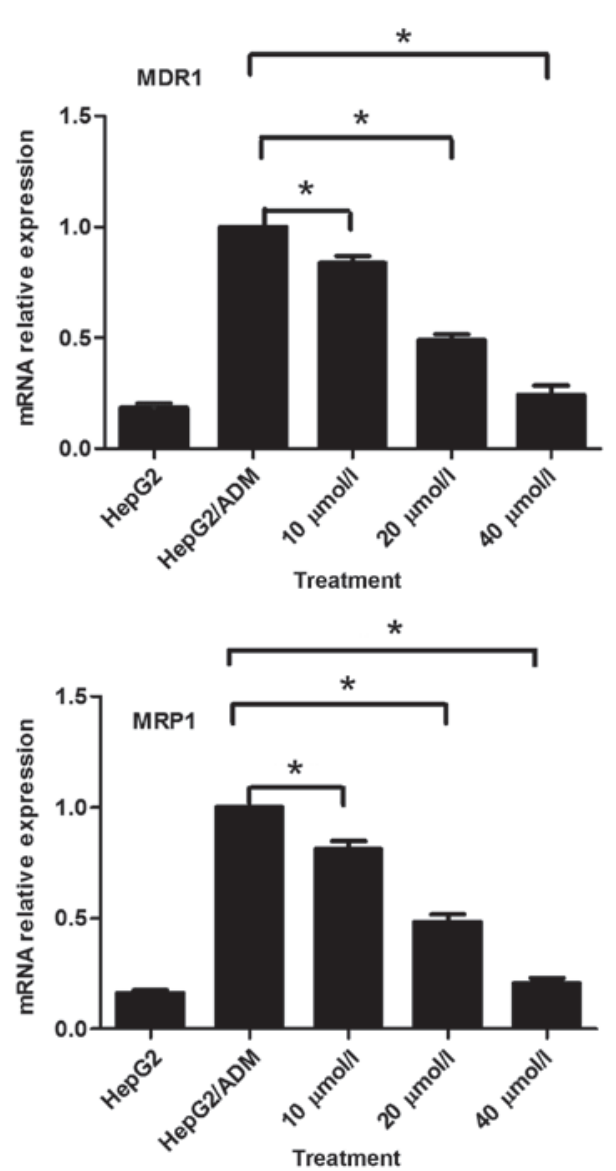

Figure 5. Reverse transcription-quantitative polymerase chain reaction of MDR1 and MRP1 expression. The expression of MDR1 and MRP1 for HepG2/ADM were increased 5.37- and 6.14-fold compared with that of HepG2 cells, respectively. The expression of MDR1 and MRP1 HepG2/ADM cells was significantly decreased following treatment with U0126 in dose-dependent manner. Results are presented as the mean \pm standard deviation $(\mathrm{n}=3)$. $\mathrm{P}<0.05$ following one-way analysis of variance. MDR 1 , multidrug resistance 1; MRP1, multidrug resistance-associated protein 1 .

conform to MDR standards (18) and may therefore be used to investigate reversion of MDR.

Currently, research into the mechanism of MDR in tumor cells has focused on the proteins P-gp, MRP and LRP, and the enzymes glutathione transferase and DNA topoisomerase (15). In the present study, P-gp and MRP1 protein expression was demonstrated to be increased in drug-resistant HepG2/ADM cells compared with drug-sensitive HepG2 

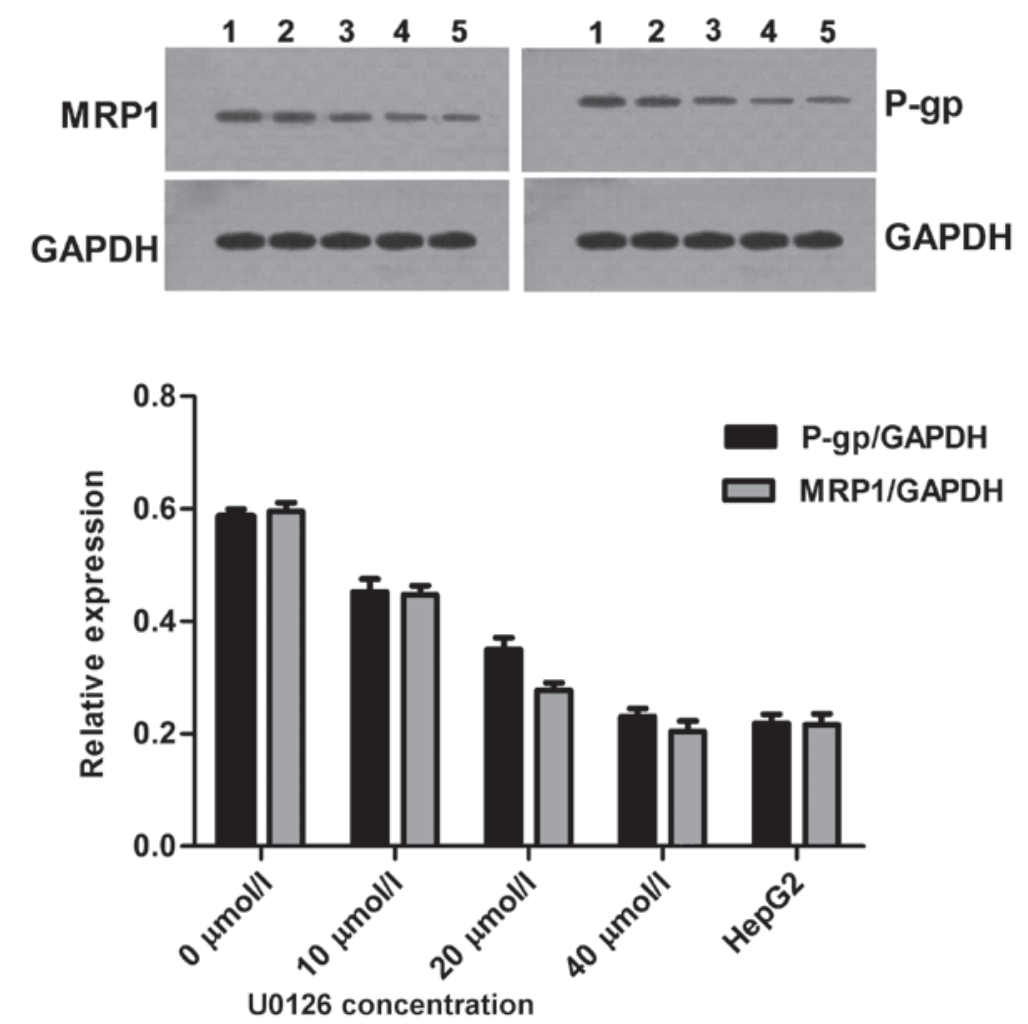

Figure 6. Protein expression of P-gp and MRP1 in HepG2 and HepG2/ADM cells determined by quantification of western blots. Lane 1, HepG2/ADM cells $+0.4 \mu \mathrm{mol} / 1 \mathrm{ADM}$; lane 2, HepG2/ADM cells $+0.4 \mu \mathrm{mol} / 1 \mathrm{ADM}+10 \mu \mathrm{mol} / 1 \mathrm{U} 0126$; lane 3, HepG2/ADM cells $+0.4 \mu \mathrm{mol} / 1 \mathrm{ADM}+20 \mu \mathrm{mol} / 1 \mathrm{U} 0126$; lane 4 , HepG2/ADM cells $+0.4 \mu \mathrm{mol} / 1 \mathrm{ADM}+40 \mu \mathrm{mol} / 1 \mathrm{U} 0126$; lane 5, HepG2 cells. P-gp expression was increased 2.68-fold in HepG2/ADM cells compared with that in HepG2 cells. However, MRP1 expression was increased 2.76-fold in HepG2/ADM cells compared with that in HepG2 cells. The protein expression of P-gp and MRP1 in HepG2/ADM cells treated with different concentrations of U0126 were decreased significantly compared with control HepG2/ADM cells. P-gp, P-glycoprotein; MRP1, multidrug resistance-associated protein 1; ADM, Adriamycin.

parental cells, suggesting that typically used chemotherapeutic drugs may lead to MDR in hepatoma cells. This is similar to the study by Chaudhary and Roninson (19), which demonstrated that acquired resistance may occur through the induction of short-term chemotherapy and is retained for $\sim 6$ weeks (19). P-gp and MRP1 belong to the ABC membrane transport protein superfamily. These proteins may mediate the excretion of various antitumor drugs, resulting in the concentration of the drugs being lower than the effective concentration required to limit the proliferation of the tumor cell; conversely, these proteins also have an effect on the distribution of chemotherapeutic drugs in tumor cells, leading to the acquisition of MDR by tumor cells (20). Therefore, the concentration of chemotherapeutic drugs in tumor cells may be improved by adjusting the expression of $\mathrm{ABC}$ proteins to enhance anti-tumor activity.

MAPKs are present in the majority of cell types, and transmit extracellular stimulating signals to regulate cell growth, differentiation, proliferation, apoptosis and other biological effects. A number of parallel MAPK signaling pathways have been identified, among which the ERK/MAPK signaling pathway is the typical signal regulation and control pathway, and the c-Jun N-terminal kinase/stress-activated protein kinase and $\mathrm{p} 38^{\mathrm{MAPK}}$ pathway frequently modulates the biological effect under stress conditions (12). Hu et al (21) demonstrated an increased effect of chemotherapeutic drugs on H460 non-small cell lung cancer cells following suppression of the ERK/MAPK signaling pathway. Eum et al (22) demonstrated that the Raf/MEK/ERK signaling pathway inhibitor PLX4720 may reverse the drug resistance of NIH3T3/MDR cells by decreasing the expression of P-gp, which is consistent with the results of the present study. Using the MEK inhibitor U0126 in HepG2/ADM cells, it was demonstrated that the treatment may inhibit the proliferation of HepG2/ADM cells and promote apoptosis; furthermore, with increasing concentrations, U0126 was able to decrease the mRNA and protein expression of P-gp and MRP1, suggesting that the drug resistance of HepG2/ADM cells may be reversed. However, further research is required to identify the specific association and underlying molecular mechanism.

The association between MAPK and MDR protein remains unclear, and the underlying molecular mechanism of MDR regulation by MAPK also remains unclear. Lee et al (23) reported that another signaling pathway [the phosphoinositide 3-kinase (PI3K)/protein kinase B (AKT) signaling pathway] is associated with MDR: PI3K activation in prostate cancer drug-resistant cell was demonstrated to generate MDR by increasing the expression of MRP1. In leukemic drug-resistant cells, the drug resistance of MRP1 was decreased by inhibiting the PI3K/AKT signal transmission pathway (24); however, whether they are associated remains unclear. The regulation and underlying molecular mechanism of MDR is complicated, which may result from combined regulation and control of numerous signaling pathways.

In conclusion, the HepG2/ADM cell model of MDR was used to investigate the underlying molecular mechanism for 
resistance and to identify a drug that may overcome drug resistance. The MEK inhibitor U0126 may decrease the mRNA and protein expression levels of drug-resistant proteins, and promote apoptosis of drug-resistant cells, which provides a theoretical basis for combined chemotherapy in the treatment of liver cancer.

\section{References}

1. International Agency for Research on Cancer (IARC): GLOBOCAN 2008: Cancer Incidence and Mortality Worldwide. IARC, Lyon, 2010. https://www.iarc.fr/en/media-centre/iarcnews/2010/ globocan2008.php. Accessed May 1, 2010.

2. Jemal A, Bray F, Center MM, Ferlay J, Ward E and Forman D: Global cancer statistics. CA Cancer J Clin 61: 69-90, 2011.

3. Fang Y, Shang QL, Liu JY, Li D, Xu WZ, Teng X, Zhao HW, Fu LJ, Zhang FM and Gu HX: Prevalence of occult hepatitis B virus infection among hepatopathy patients and health people in China. J Infect 58: 383-388, 2009.

4. Al-Rawashdeh FY, Scriven P, Cameron IC, Vergani PV and Wyld L: Unfolded protein response activation contributes to chemoresistance in hepatocellular carcinoma. Eur J Gastroenterol Hepatol 22: 1099-1105, 2010.

5. Gottesman MM, Fojo T and Bates SE: Multidrug resistance in cancer: Role of ATP-dependent transporters. Nat Rev Cancer 2: 48-58, 2002.

6. Lee $\mathrm{CH}$ : Reversing agents for ATP-binding cassette drug transporters. Methods Mol Biol 596: 325-340, 2010.

7. Anders M, Christian C, McMahon M, McCormick F and Korn WM: Inhibition of the Raf/MEK/ERK pathway up-regulates expression of the coxsackievirus and adenovirus receptor in cancer cells. Cancer Res 63: 2088-2095, 2003.

8. Viala E and Pouysségur J: Regulation of tumor cell motility by ERK mitogen-activated protein kinases. Ann N Y Acad Sci 1030 208-218, 2004

9. Wilkinson MG and Millar JB: Control of the eukaryotic cell cycle by MAP kinase signaling pathways. FASEB J 14: 2147-2157, 2000.

10. Johnson GL and Lapadat R: Mitogen-activated protein kinase pathways mediated by ERK, JNK, and p38 protein kinases. Science 298: 1911-1912, 2002.

11. Shen H, Xu W, Luo W, Zhou L, Yong W, Chen F, Wu C, Chen Q and Han X: Upregulation of mdrl gene is related to activation of the MARK/ERK signal transduction pathway and YB-1 nuclear translocation in B-cell lymphoma. Exp Hematol 39: 558-569, 2011.

12. Abrams SL, Steelman LS, Shelton JG, Wong EW, Chappell WH, Bäsecke J, Stivala F, Donia M, Nicoletti F, Libra M, et al: The Raf/MEK/ERK pathway can govern drug resistance, apoptosis and sensitivity to targeted therapy. Cell Cycle 9: 1781-1791, 2010.
13. Katayama K, Yoshioka S, Tsukahara S, Mitsuhashi J and Sugimoto Y: Inhibition of the mitogen-activated protein kinase pathway results in the down-regulation of P-glycoprotein. Mol Cancer Ther 6: 2092-2102, 2007.

14. Berger W, Setinek U, Hollaus P, Zidek T, Steiner E, Elbling L, Cantonati H, Attems J, Gsur A and Micksche M: Multidrug resistance markers P-glycoprotein, multidrug resistance protein1, and lung resistance protein in non-small cell lung cancer: Prognostic implications. J Cancer Res Clin Oncol 131: 355-363, 2005.

15. Wang J, Zhang J, Zhang L, Zhao L, Fan S, Yang Z, Gao F, Kong Y, Xiao GG and Wang Q: Expression of P-gp, MRP, LRP, GST- $\pi$ and TopoII $\alpha$ and intrinsic resistance in human lung cancer cell lines. Oncol Rep 26: 1081-1089, 2011.

16. Tivnan A, Zakaria Z, O'Leary C, Kögel D, Pokorny JL, Sarkaria JN and Prehn JH: Inhibition of multidrug resistance protein1 (MRP1) improves chemotherapy drug response in primary and recurrent glioblastoma multiforme. Front Neurosci 9: 218, 2015

17. Stavrovskaya AA: Cellular mechanisms of multidrug resistance of tumor cells. Biochemistry (Mosc) 65: 95-106, 2000

18. Snow K and Judd W: Characterisation of Adriamycin- and amsacrine-resistant human leukaemic T cell lines. Br J Cancer 63: 17-28, 1991

19. Chaudhary PM and Roninson IB: Induction of multidrug resistance in human cells by transient exposure to different chemotherapeutic drugs. J Natl Cancer Inst 85: 632-639, 1993.

20. Wang XK and Fu LW: Interaction of tyrosine kinase inhibitors with the MDR-related ABC transporter proteins. Curr Drug Metab 11: 618-628, 2010.

21. Hu Y, Bally M, Dragowska WH and Mayer L: Inhibition of mitogen-activated protein kinase/extracellular signal-regulated kinase enhances chemotherapeutic effects on H460 human non-small cell lung cancer cells through activation of apoptosis. Mol Cancer Ther 2: 641-649, 2003.

22. Eum KH, Ahn SK, Kang H and Lee M: Differential inhibitory effects of two Raf-targeting drugs, sorafenib and PLX4720, on the growth of multidrug-resistant cells. Mol Cell Biochem 372: 65-74, 2013.

23. Lee JT Jr, Steelman LS and McCubrey JA: Phosphatidylinositol 3'-kinase activation leads to multidrug resistance protein-1 expression and subsequent chemoresistance in advanced prostate cancer cell. Cancer Res 64: 8397-8404, 2004.

24. Tazzari PL, Cappellini A, Ricci F, Evangelisti C, Papa V, Grafone T, Martinelli G, Conte R, Cocco L, McCubrey JA and Martelli AM: Multidrug resistance-associated protein 1 expression is under the control of the phosphoinositide 3 kinase/Akt signal transduction network in human acute myelogenous leukemia blasts. Leukemia 21: 427-438, 2007. 